\title{
Removal of Iron and Copper lons from the Liquid Phase by Modified Polymeric Membranes
}

\author{
Joanna Krason ${ }^{1} \cdot$ Robert Pietrzak ${ }^{1}$ \\ Published online: 19 February 2018 \\ (c) The Author(s) 2018. This article is an open access publication
}

\begin{abstract}
Preparation of polymeric membranes based on cellulose acetate and modified by adding different amounts of a pore generating agent, which was polyvinylpyrrolidone (PVP), is presented and potential application of the membranes obtained for the removal of iron and copper ions from liquid phase is examined. Addition of various amounts of PVP has an impact on the physical (porosity, equilibrium water content and permeability) and chemical (content of the surface oxygen group, $\mathrm{pH}$ ) properties of the membranes obtained. Filtration of iron and cupper solution leads to significant changes in the total content of surface oxygen groups, however the acidic oxygen groups remain dominant. With the content of PVP increasing within a certain range, the membrane permeability is improved. The membranes obtained show higher efficiency in removal of $\mathrm{Fe}^{3+}$ than $\mathrm{Cu}^{2+}$ ions, but with increasing content of PVP the efficiency in iron ions removal decreases and the total filtration resistance decreases after filtration of iron ions solutions.
\end{abstract}

Keywords Cellulose acetate membrane · Phase inversion · Copper and iron ions removal · Physical and chemical properties $\cdot$ Surface chemistry

\section{Introduction}

Dynamical development of economy and industry taking place over the last few decades has brought about the need of searching for more effective methods for dealing with increasing industrial waste and environmental pollution. Not only the contaminants generated in the process of production of certain materials and products, but also those forming as side effects of using the ready products must be removed. One of the most promising methods is that based on the use of membrane processes. These processes have been used in chemical industry (including petrochemical), pharmaceutical industry, production of food and drinks [1-3]. Numerous membrane installations are used for gases separation in chemical and petrochemical industry. They permit separation of volatile hydrocarbons from the stream of air or separate monomers from the stream of exhaust gases in

Robert Pietrzak

pietrob@amu.edu.pl

1 Laboratory of Applied Chemistry, Faculty of Chemistry, Adam Mickiewicz University in Poznań, Umultowska 89b, 61-614 Poznań, Poland polymeric installations and direct them to the next stage of the process [4-7].

Membrane processes do not require dosing of chemical agents and do not involve transformations of pollutants, which saves resources, energy and labor, permitting rational water management and utilization of industrial waste. Modern membranes are effective in removal of pollutants from liquid phase [8-11], which is important from the point of view of the environment protection, and offers a possibility of recovery of valuable components, which is economically important.

A pressing problem is the pollution of water environment with heavy metals. Their increased content deteriorates the quality of drinking water and is hazardous to human health $[12,13]$. Many methods have been proposed to curb their amount or to eliminate them from water, like e.g. extraction with solvents, coagulation and flocculation, electrocoagulation, adsorption on various materials or membrane separation. The latter method has been increasingly often applied because of high efficiency and low cost of the process [14-17]. There is one more important benefit from using membrane processes, by appropriate membrane modification it is possible to obtain membranes highly selective towards certain compounds [18]. 
The membranes used for removal of pollutants from liquid phase are mainly made of polymers such as polypropylene, polysulfone, polyethersulfone, polycarbonates, polyamides and cellulose esters, including cellulose acetate [19-21]. The membranes made of cellulose acetate have found wide application in the pressure separation techniques, e.g. ultrafiltration or reversed osmosis $[22,23]$. The efficiency of separation is determined not only by the type of polymer of which a given membrane is made, but also by the type of additive polymers stimulating pore generation [24]. A well-known and widely used method for the production of synthetic membranes is the inversion of phases, in which the scattering phase becomes the scattered phase and vice versa [25-27]. A mixture of the polymer, solvent and pore-generating agent is spread out on a substrate, e.g. glass, by a special knife, then the solvent is evaporated and the polymer is immersed in a bath with a non-solvent, e.g. distilled water [28-30].

The aim of this paper was the synthesis and characterization of membranes based on cellulose acetate and containing different amounts of pore generating agent (polyvinyl- pyrrolidone, PVP), in particular, determination of their ability to remove copper and iron ions from liquid phase.

\section{Experimental}

\section{Materials}

Cellulose acetate (CA, average $\left.M_{n} \sim 30,000\right)$ and polyvinylpyrrolidone (PVP, average wt\% 10,000) were purchased from Sigma Aldrich. CA was used as a membrane polymer and PVP was used as a pore-generating agent. $N, N$-dimethylformamide (DMF, > 99.8\%) was purchased from Avantor Performance Materials Poland S.A. and used as a solvent.

The sources of copper and iron ions were copper(II) sulfate $\left(\mathrm{CuSO}_{4} \cdot 5 \mathrm{H}_{2} \mathrm{O}\right)$ and ammonium iron(III) sulfate $\left(\mathrm{NH}_{4} \mathrm{Fe}\left(\mathrm{SO}_{4}\right)_{2} \cdot 12 \mathrm{H}_{2} \mathrm{O}\right)$, from Avantor Performance Materials Poland S.A.

\section{Preparation of CA Membranes}

At the first stage of membrane preparation, the so-called membrane film was obtained by mixing appropriate amounts of reagents. Glass flasks were charged with 1, 2, 3 or $4 \mathrm{wt} \%$ of PVP and $85,84,83$ or $82 \mathrm{wt} \%$ of DMF. Then each flask was placed on a magnetic stirrer and stirred at 600 rev./ min. Then CA was added in portions, to the final content of $14 \mathrm{wt} \%$. and then the flask was placed in a water bath and heated at $60{ }^{\circ} \mathrm{C}$ for $24 \mathrm{~h}$, upon continuous stirring to release bubbles. After careful stirring of the components, the solutions were left on the magnetic stirrer to cool down to room temperature and then the films were prepared by spreading the contents on a glass plate using a special knife Elcometer 3580 made by Sterlitech Corporation and left for $40 \mathrm{~s}$ to evaporate the solvent. The plate with the film was immersed in a coagulation bath of deionized water, to obtain membranes in the form of plane sheets of $300 \mu \mathrm{m}$ in thickness. The particular samples were labelled as CA14 PVP1, CA14 PVP2, CA14 PVP3 and CA14 PVP4. The membranes obtained were washed for additional $24 \mathrm{~h}$ using deionized water bath conditioned at $25^{\circ} \mathrm{C}$.

\section{Membrane Structure Characterization}

\section{Porosity and Equilibrium Water Content}

Porosity of the membranes obtained was characterized on the basis of the membrane mass loss after drying. The membranes taken out of the deionized water were weighted and then placed in a drier at $60{ }^{\circ} \mathrm{C}$ for $24 \mathrm{~h}$. After this time the membranes were weighted again and their porosity was calculated from Eq. (1):

$\varepsilon=\frac{W_{w}-W_{d}}{\rho \times v} \cdot 100 \%$

where $\mathrm{W}_{\mathrm{w}}$ is the mass of a wet membrane sample, $\mathrm{W}_{\mathrm{d}}$ is the membrane mass at dry state, $\rho$ is the pure water density and $\mathrm{v}$ is the membrane volume at wet state.

The equilibrium water content (EWC) was determined from Eq. (2):

$E W C=\frac{W_{w}-W_{d}}{W_{w}} \times 100 \%$

\section{Contact Angle}

The contact angle between water and membrane surface was directly measured using a measuring instrument G10, KRUSS, Germany. Deionized water was used as a probe liquid (sessile drop technique) in all measurements. The contact angle between water and membrane was measured five times at random places for each sample and then the average was calculated.

\section{Surface Oxygen Groups}

The surface oxygen groups were characterized using potentiometric titration experiments with the help of 809 Titrando, Metrohm. The instrument was set in the mode of equilibrium $\mathrm{pH}$ measurement and recording. Membranes in the amount of about $0.05 \mathrm{~g}$ in $50 \mathrm{~mL} 0.01 \mathrm{M} \mathrm{NaNO}_{3}$ were placed in a vessel thermostated at $25^{\circ} \mathrm{C}$ and equilibrated for $1 \mathrm{~h}$ with the electrolyte solution. The suspension was stirred throughout the measurements. The volumetric standards $\mathrm{NaOH}(0.1 \mathrm{M})$ or $\mathrm{HCl}(0.1 \mathrm{M})$ were used as titrants [31]. 


\section{Membrane Performance Characterization}

Water permeability of the membranes prepared was measured in a stainless steel membrane module, for the effective membrane area of $19.6 \mathrm{~cm}^{2}$. The pure water flux was measured at 3 bar, $23 \pm 1{ }^{\circ} \mathrm{C}$ and $0.22 \mathrm{~m} / \mathrm{s}$ cross-flow velocity. The pure water flux was calculated from Eq. (3):

$J_{w}=\frac{V}{A \times \Delta t}$

where $J_{w}\left(L /\left(m^{2} h\right)\right)$ is the pure water flux, V (L) is the volume of permeated water, $\mathrm{A}\left(\mathrm{m}^{2}\right)$ is the effective membrane area and $\Delta \mathrm{t}(\mathrm{h})$ is the permeation time.

The experiments were conducted with compressed nitrogen gas and iron or copper solutions of different initial concentrations $(12$ or $20 \mathrm{mg} / \mathrm{L}$ for iron solution and 800 or $1000 \mathrm{mg} / \mathrm{L}$ for solution of copper). All measurements were carried at $3 \mathrm{bar}$, in triplicate. The final concentration of iron or copper in the solution was determined using a double beam UV-Vis spectrophotometer Varian Cary 100 Bio. Measurements were made at $487 \mathrm{~nm}$ wavelength for iron solutions and at $620 \mathrm{~nm}$ wavelength for copper solutions. The amount of metal ions removed (in \%) was found from the following equation:

$\% R=\left(1-\frac{C_{p}}{C_{f}}\right) \times 100$

where $\mathrm{C}_{\mathrm{p}}$ is the concentration in the permeate $(\mathrm{mg} / \mathrm{L})$ and $\mathrm{C}_{\mathrm{f}}$ is the concentration in the feed solution $(\mathrm{mg} / \mathrm{L})$.

Membrane resistance was calculated from Eq. (5):

$J=\frac{\Delta P}{\mu R_{t}}$

where $\mathrm{J}$ is the permeate flux $\left[\mathrm{L} /\left(\mathrm{m}^{2} \mathrm{~h}\right)\right], \Delta \mathrm{P}$ is the transmembrane pressure, $\mu$ is the dynamic viscosity of permeate $[\mathrm{Pa} \cdot \mathrm{s}]$, and $\mathrm{R}_{\mathrm{t}}$ is the total filtration resistance $[\mathrm{m} / \mathrm{s}]$. The total filtration resistance comprises three terms: resistance of the membrane $\left(\mathrm{R}_{\mathrm{m}}\right)$, resistance of the pores that undergo blocking $\left(\mathrm{R}_{\mathrm{p}}\right)$ and that of the filtration cake $\left(\mathrm{R}_{\mathrm{c}}\right)$. The total filtration resistance was calculated from Eq. (6):

$R_{t}=R_{m}+R_{p}+R_{c}$

The flux recovery ratio (FRR) was studied as follows. Firstly, pure water flux of the membrane $J_{w 1}\left(L /\left(m^{2} h\right)\right)$ was tested at 3 bar. After filtration of metal ions solution, the membrane was rinsed with deionized water for $10 \mathrm{~min}$ and then pure water flux of the membrane $\mathrm{J}_{\mathrm{w} 2}\left(\mathrm{~L} /\left(\mathrm{m}^{2} \mathrm{~h}\right)\right)$ was measured. The FRR was calculated from Eq. (7):

$\operatorname{FRR}(\%)=\frac{J_{w 2}}{J_{w 1}} \times 100 \%$
Table 1 Porosity $(\varepsilon)$, equilibrium water content (EWC) and contact angle of membranes investigated

\begin{tabular}{llll}
\hline Membrane & $\varepsilon(\%)$ & EWC $(\%)$ & Contact angle \\
\hline CA14 PVP1 & 46.08 & 83.09 & $65.4 \pm 3.00$ \\
CA14 PVP2 & 43.23 & 82.50 & $52.6 \pm 7.68$ \\
CA14 PVP3 & 52.93 & 83.40 & $53.5 \pm 8.34$ \\
CA14 PVP4 & 52.93 & 83.70 & $54.4 \pm 2.98$ \\
\hline
\end{tabular}

Table 2 Acidic and basic properties of membranes [mmol/g]

\begin{tabular}{llll}
\hline Membrane & Acidic groups & Basic groups & $\begin{array}{l}\text { Total content } \\
\text { of oxygen } \\
\text { groups }\end{array}$ \\
\hline CA14 PVP1 & 4.58 & 2.61 & 7.19 \\
CA14 PVP2 & 4.51 & 1.72 & 6.23 \\
CA14 PVP3 & 4.60 & 1.70 & 6.30 \\
CA14 PVP4 & 4.42 & 1.65 & 6.07 \\
\hline
\end{tabular}

\section{Results and Discussion}

\section{Characterization of Membranes}

Table 1 presents the structural parameters and contact angles determined for the series of membranes studied. Analysis of these results reveals that the values of these parameters are not directly related to the content of PVP in the membrane. The porosity of membrane CA14 PVP1 is $46.08 \%$, while that for the membrane with $2 \%$ addition of PVP is $43.23 \%$. Much higher values were obtained for membranes CA14 PVP3 and CA14 PVP4, of 52.93\% for each of them. The differences in these values follow from the fact that pore distribution in the membrane is inhomogeneous. All membranes were characterized by similar values of EWC; for CA14 PVP1 it was $83.09 \%$, for CA14 PVP2 it was $82.50 \%$, for CA14 PVP3 and CA14 PVP4 the values of EWC were 83.40 and $83.70 \%$, respectively. The highest contact angle value of 65.4 was found for the membrane with the lowest content of PVP. For the other membranes, the contact angle values were similar 52.6 for CA14 PVP2, 53.5 for CA14 PVP3 and 54.4 for CA14PVP4 with the highest content of PVP, which can be related to the hydrophilic properties of this polymer.

\section{Membrane Performance}

Table 2 presents the contents of acidic and basic oxygen groups on the surfaces of the membranes studied. The amounts of acidic oxygen groups are similar on the 
surfaces of all membranes, on CA14 PVP1 their content was $4.58 \mathrm{mmol} / \mathrm{g}$, on CA14 PVP2 - $4.51 \mathrm{mmol} / \mathrm{g}$, on CA14 PVP3 and CA14 PVP4 their amounts were 4.60 and $4.42 \mathrm{mmol} / \mathrm{g}$, respectively. The acidic surface oxygen groups were dominant on all membranes. The basic oxygen groups were present in much smaller amounts. They occurred in the greatest amount of $2.61 \mathrm{mmol} / \mathrm{g}$ on the surface of CA14 PVP1, while on the surfaces of the other membranes their amounts were smaller, varying from 1.65 to $1.72 \mathrm{mmol} / \mathrm{g}$. Also the total content of oxygen groups on the surface of the membranes was the greatest, $7.19 \mathrm{mmol} / \mathrm{g}$, on CA14PVP1 with the lowest content of PVP. The total content of oxygen surface groups on the other three membranes was $6.23 \mathrm{mmol} / \mathrm{g}$ for CA14 PVP2, $6.30 \mathrm{mmol} / \mathrm{g}$ for CA14 PVP3 and $6.07 \mathrm{mmol} / \mathrm{g}$ for CA14PVP4 with the greatest content of PVP.

The data on permeability of CA14 series membranes are presented in Fig. 1. The highest permeability after the filtration of iron solution of $165.58 \mathrm{~L} / \mathrm{m}^{2} \mathrm{~h}$, is found for the membrane with $3 \mathrm{wt} \%$ of PVP, which is interpreted as related to the fact that this membrane shows the highest porosity. For the membrane with $4 \mathrm{wt} \%$ of PVP, the permeability of pure water flux is a little lower, $161.71 \mathrm{~L} / \mathrm{m}^{2} \mathrm{~h}$. For membranes CA14 PVP1 and CA14 PVP2 the permeability increased with increasing content of PVP and took the values 54.95 and $78.25 \mathrm{~L} / \mathrm{m}^{2} \mathrm{~h}$. After the filtration of copper ions solution, the highest permeability was measured for membrane CA14 PVP3 $\left(168.74 \mathrm{~L} / \mathrm{m}^{2} \mathrm{~h}\right)$. For sample CA14 PVP4 the permeability was $138.45 \mathrm{~L} / \mathrm{m}^{2} \mathrm{~h}$, while for the other two membranes the permeability was much lower $60.90 \mathrm{~L} / \mathrm{m}^{2} \mathrm{~h}$.for CA14 PVP1 and $35.22 \mathrm{~L} / \mathrm{m} 2 \mathrm{~h}$ for CA14 PVP2.

Figure 2 presents results illustrating the efficiency of iron ions removal from the solutions of different iron concentrations by the membranes synthesized with different contents of PVP. Irrespectively of the initial concentration of the solution, the most effective in iron ions removal were the membranes with the lowest contents of PVP, which removed 53 and $47 \%$ of iron ions. High capacity for iron ions removal

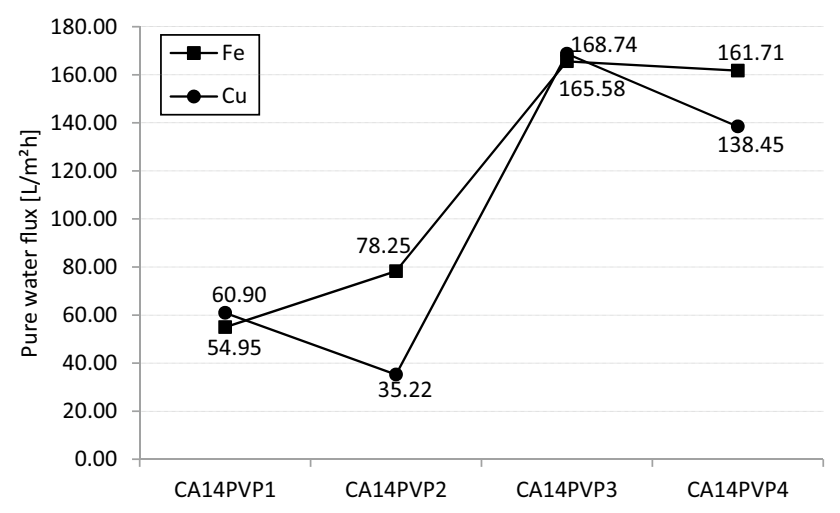

Fig. 1 Pure water flux of the membranes studied

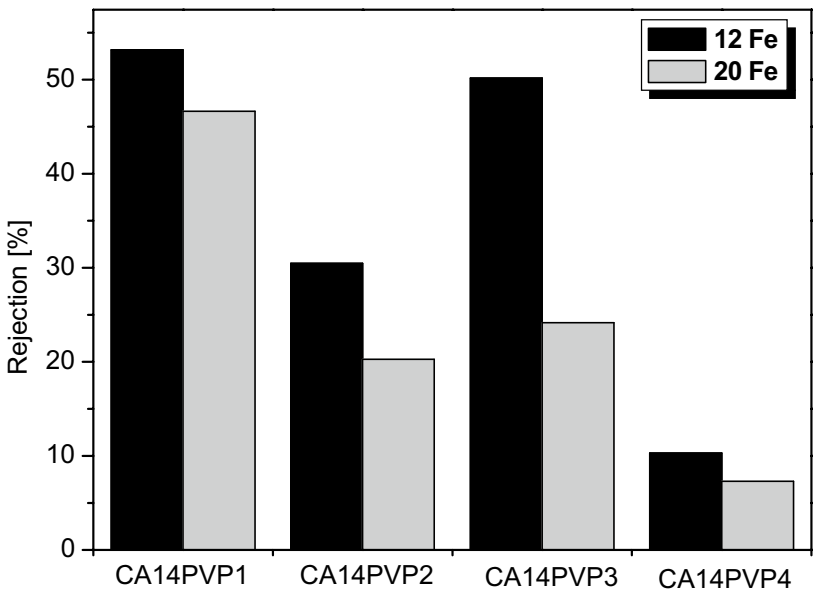

Fig. 2 Iron rejection of CA14 series membranes after filtration of an iron solution

(50\%) was noted for membrane CA14 PVP3 when used for filtration of iron ions from the solution of $12 \mathrm{mg} / \mathrm{L}$. The capacities for iron removal observed for the other two membranes were much smaller, of 30 and $10 \%$. When filtration was performed from the solution of $20 \mathrm{mg}$ iron ions/L, only sample CA14 PVP1 showed relatively good capacity for ion ions removal, while the other ones containing 2, 3 and $4 \mathrm{wt} \%$ of PVP were much less effective and their capacities for ion ions removal were 20, 24 and $7 \%$. The most efficient in iron ions removal were the membranes with the lowest content of PVP, while the least efficient was that with the highest content of this pore generating polymer. It means that with increasing amount of pores the membranes lose the ability of effective removal of iron ions. The results also show that irrespective of the type of membrane the most effectively purified are the solutions of lower concentration of $\mathrm{Fe}^{3+}$ ions.

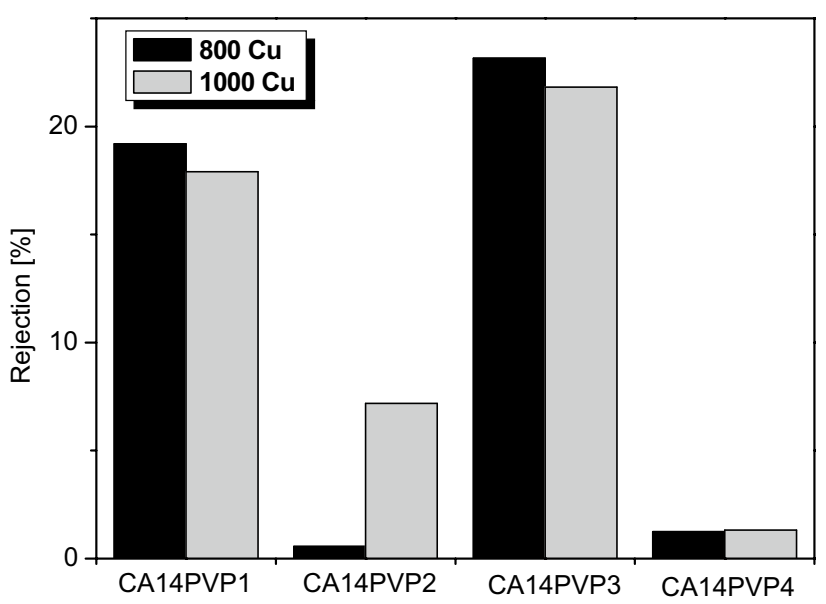

Fig. 3 Copper rejection of CA14 series membranes after filtration of a copper solution 
Figure 3 presents the data characterizing sorption abilities of the membranes used for filtration of copper ions. The highest sorption capacity was obtained for membrane CA14 PVP3 which removed 23\% of copper ions from a solution of the concentration $800 \mathrm{mg} / \mathrm{L}$ and $22 \%$ of copper ions from a solution of the concentration $1000 \mathrm{mg} / \mathrm{L}$. The sorption capacity of membrane CA14 PVP1 was a bit lower, it removed $19 \%$ of copper ions from the solution of the initial concentration $800 \mathrm{mg} / \mathrm{L}$ and $18 \%$ of copper ions from the solution of $1000 \mathrm{mg} / \mathrm{L}$. Membrane CA14 PVP2 removed $7 \%$ of copper ions from the solution of $1000 \mathrm{mg} / \mathrm{L}$, while only $0.6 \%$ of copper ions from the solution of $800 \mathrm{mg} / \mathrm{L}$. Membrane CA14 PVP4 showed very low sorption capacity towards copper ions, within $1 \%$, irrespective of the solution initial concentration.

Figure 4 presents the degree of recovery of the membranes after filtrations of iron and copper ions, expressed in terms of the flux recovery rate (FRR). The value of this parameter informs about the membranes efficiency and about the theoretical possibility of their reuse for the filtration of solutions. After filtration of iron solutions, the values of FRR were the highest for CA14 PVP1 and CA14 PVP2, (Fig. 4a). FRR for CA14 PVP4 was lower and for CA14 PVP3 it was the lowest. After filtration of copper ions,
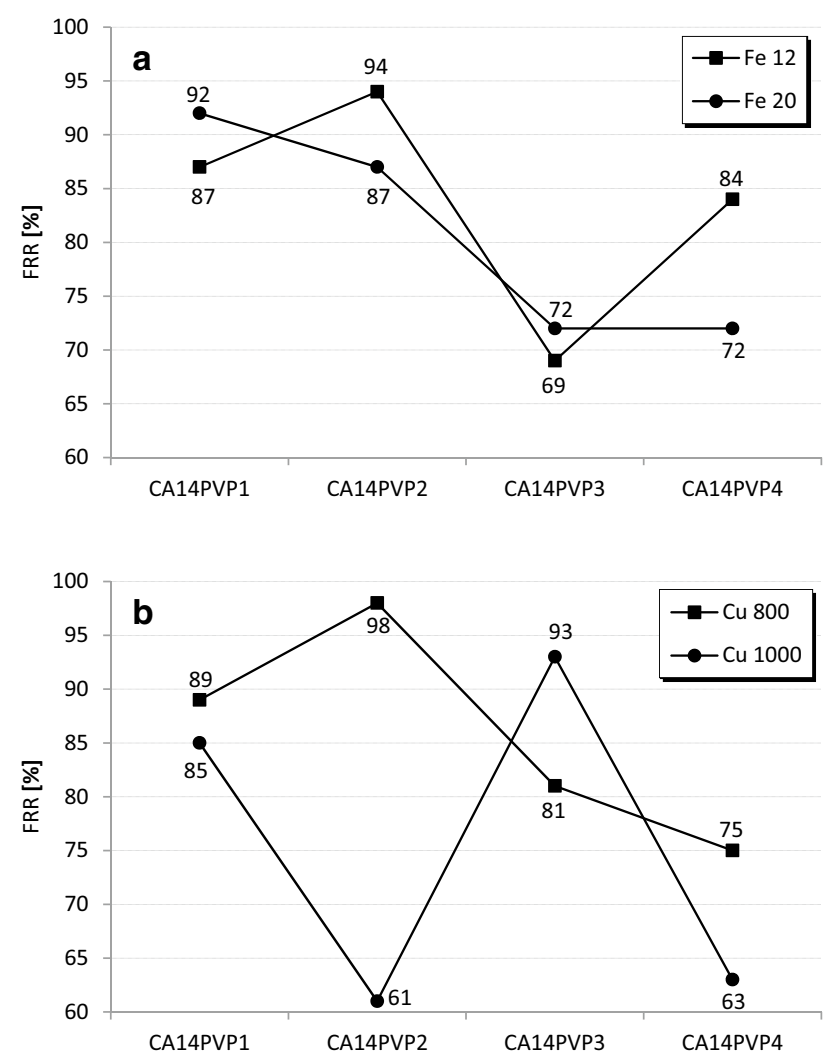

Fig. 4 Flux recovery ratio of membranes studied after filtration of $\mathbf{a}$ iron and $\mathbf{b}$ copper solution the highest FRR values were measured for CA14 PVP1 and CA14 PVP3 (Fig. 4b), while the lowest FRR was obtained for CA14 PVP4. No effect of the PVP content in the membrane on the FRR value was noted. When filtering copper solutions, for the majority of samples higher FRR values were obtained for the samples of lower initial concentration. The values evidence high effectiveness of the membranes studied. Moreover, the FRR values indicate the theoretical possibility of reuse of the membranes for removal of liquid phase pollutants. High FRR values mean that after filtration the membranes are effectively cleaned so the blocking of their pores and surfaces by the removed compounds is relatively small.

Table 3 presents the data on filtration resistance of the membranes used for the filtration of iron ions solutions. As follows from these data, the total filtration resistance Rt decreases with increasing content of the pore generating agent PVP, so from CA14 PVP1 to CA14 PVP4. The value of $R_{t}$ for CA14 PVP1 was $8.37 \times 10^{13} \mathrm{~L} / \mathrm{m}^{2} \mathrm{~h}$, it was twice lower, $4.08 \times 10^{13} \mathrm{~L} / \mathrm{m}^{2} \mathrm{~h}$ for CA14 PVP2, and for CA14 PVP3 and CA14 PVP4 it was 2.34 and $2.31 \times 10^{13} \mathrm{~L} /$ $\mathrm{m}^{2} \mathrm{~h}$, respectively. The values of individual components of the total filtration resistance also decreased with increasing content of PVP, the exception was the membrane resistance which was a bit higher for CA14 PVP4 than for CA14 PVP3.

The values of different types of resistance determined for the membranes studied after filtration of copper ions are collected in Table 4. The highest components of resistance were measured for CA14 PVP2 whose total resistance is $12.82 \times 10^{13} \mathrm{~L} / \mathrm{m}^{2} \mathrm{~h}$ and is much higher than the values obtained for the other membranes. The components of total resistance for this membrane were as follows: membrane resistance $2.99 \times 10^{13} \mathrm{~L} / \mathrm{m}^{2} \mathrm{~h}$, pore resistance $4.94 \times 10^{13} \mathrm{~L} /$ $\mathrm{m}^{2} \mathrm{~h}$ and cake formation resistance $4.89 \times 10^{13} \mathrm{~L} / \mathrm{m}^{2} \mathrm{~h}$.

Table 3 Filtration resistance of different membranes for iron solution

\begin{tabular}{lllll}
\hline Membrane & $\mathrm{R}_{\mathrm{m}}\left(\times 10^{13}\right)$ & $\mathrm{R}_{\mathrm{p}}\left(\times 10^{13}\right)$ & $\mathrm{R}_{\mathrm{c}}\left(\times 10^{13}\right)$ & $\mathrm{R}_{\mathrm{t}}\left(\times 10^{13}\right)$ \\
\hline CA14 PVP1 & 2.70 & 2.93 & 2.74 & 8.37 \\
CA14 PVP2 & 1.25 & 1.43 & 1.40 & 4.08 \\
CA14 PVP3 & 0.64 & 0.89 & 0.81 & 2.34 \\
CA14 PVP4 & 0.68 & 0.85 & 0.78 & 2.31 \\
\hline
\end{tabular}

Table 4 Filtration resistance of different membranes for copper solution

\begin{tabular}{lllll}
\hline Membrane & $\mathrm{R}_{\mathrm{m}}\left(\times 10^{13}\right)$ & $\mathrm{R}_{\mathrm{p}}\left(\times 10^{13}\right)$ & $\mathrm{R}_{\mathrm{c}}\left(\times 10^{13}\right)$ & $\mathrm{R}_{\mathrm{t}}\left(\times 10^{13}\right)$ \\
\hline CA14 PVP1 & 1.64 & 1.91 & 1.93 & 5.48 \\
CA14 PVP2 & 2.99 & 4.94 & 4.89 & 12.82 \\
CA14 PVP3 & 0.63 & 0.67 & 0.63 & 1.93 \\
CA14 PVP4 & 0.68 & 1.07 & 0.99 & 2.74 \\
\hline
\end{tabular}


Almost twice lower total resistance of $5.48 \times 10^{13} \mathrm{~L} / \mathrm{m}^{2} \mathrm{~h}$ was measured for CA14 PVP1, while for CA14 PVP3 and CA14 PVP4 the values of total resistance were much smaller, 1.93 and $2.74 \times 10^{13} \mathrm{~L} / \mathrm{m}^{2} \mathrm{~h}$, respectively. It should be pointed out that after filtration of iron and copper ion solutions, the values of pore resistance and cake formation resistance are similar for all membranes and the membrane resistance brings the lowest contribution to the total resistance.

\section{Conclusions}

Analysis of the above presented results permits concluding that the amount of the pore generating polymer, PVP, used in the process of membrane syntheses, has a significant influence on the properties of membranes based on cellulose acetate. The addition of PVP causes an increase in porosity, equilibrium water content and is responsible for the fact that acidic oxygen groups dominate on the surfaces of the membranes. The content of PVP has a favorable effect on the membranes permeability. The membranes studied (CA14 PVP1-4) show better efficiency in removal of $\mathrm{Fe}^{3+}$ ions than $\mathrm{Cu}^{2+}$ ones, but with increasing content of PVP the efficiency of iron ions removal decreases. After the filtration of iron ions solutions the highest degree of recovery is observed for membranes CA14 PVP1 and CA14 PVP2, while after the filtration of copper ions solutions the highest recovery is measured for membranes CA14 PVP1 and CA14 PVP3. The total filtration resistance after the filtration of iron ions solutions decreases with increasing PVP content.

Open Access This article is distributed under the terms of the Creative Commons Attribution 4.0 International License (http://creativeco mmons.org/licenses/by/4.0/), which permits unrestricted use, distribution, and reproduction in any medium, provided you give appropriate credit to the original author(s) and the source, provide a link to the Creative Commons license, and indicate if changes were made.

\section{References}

1. Zhao W, Huang J, Fang B, Nie S, Yi N, Su B, Li H, Zhao Ch (2011) J Membrane Sci 369:258-266
2. Shin SJ, Kim JP, Kim HJ, Jeon JH, Min BR (2005) Desalination 186:1-10

3. Pabby AK, Rizvi SSH, Sastre AM (2009) Membrane separations-chemical, pharmaceutical, food, and biotechnological applications. CRC Press, Taylor \& Francis Group, LLC, New York

4. Kim JH, Park PK, Lee CH, Kwon HH (2008) J Membrane Sci 321:190-198

5. Centi G, Perathoner S (2003) Appl Catal B 41:15-29

6. Basar CA, Karagunduz A, Cakici A, Keskinler B (2004) Water Res 38:2117-2124

7. Bingol A, Ucun H, Bayhan YK, Karagunduz A, Cakici A, Keskinler B (2004) Bioresour Technol 94:245-249

8. Al-Rashdi BAM, Johnson DJ, Hilal N (2013) Desalination 315:2-17

9. Saljoughi E, Mousavi SM (2012) Sep Purif Technol 90:22-30

10. Cho BY (2005) Process Biochem 40:3314-3320

11. Ghaemi N, Madaeni SS, Daraei P, Rajabi H, Zinadini S, Alizadeh A, Heydari R, Beyzadeh M (2015) Chem Eng J 263:101-112

12. Lin SH, Juang RS (2002) J Hazard Mater 92:315-326

13. Hough RL, Breward N, Young SD, Crout NMJ, Tye AM, Moir AM, Thornton I (2004) Environ Health Persp 112:215-221

14. Wiśniewska M, Bogatyrov V, Ostolska I, Szewczuk-Karpisz K, Terpiłowski K, Nosal-Wiercińska A (2016) Adsorption 22:417-423

15. Ostolska I, Wiśniewska M, Nosal-Wiercińska A, Szabelska A, Gołębiowska B (2016) Colloids Surf A Physicochem Eng Asp 488:138-144

16. Wiśniewska M, Chibowski $\mathrm{S}$, Urban T, Nosal-Wiercińska A, Gołębiowska B, Szabelska A (2017) Mater Lett 205:32-35

17. Kee CM, Idris A (2012) J Ind Eng Chem 18:2115-2123

18. Stajčić A, Nastasović A, Stajić-Trošić J, Marković J, Onjia A, Radovanović F (2015) J Environ Chem Eng 3:453-461

19. Mulder M (1996) Basic principles of membrane technology. Kluwer Academic Publisher, Dordrecht

20. Zhao C, Xue J, Ran F, Sun S (2013) Prog Mater Sci 58:76-150

21. Dasgupta J, Chakraborty S, Sikder J, Kumar R, Pal D, Curcio S, Drioli E (2014) Sep Purif Technol 133:55-68

22. Ferjani E, Ellouze E, Ben Amar R (2005) Desalination 177:43-49

23. Fischer S, Thummler K, Volkert B, Hettrich K, Schmidt I, Fischer K (2008) Macromol Sym 262:89-96

24. Jasiewicz K, Pietrzak R (2013) Chem Eng J 228:449-454

25. Saljoughi E, Mohammadi T (2009) Desalination 249:850-854

26. Kim JH, Lee KH (1998) J Membrane Sci 138:153-163

27. García-Ivars J, Alcaina-Miranda M-I, Iborra-Clar MI, MendozaRoca JA, Pastor-Alcañiz L (2014) Sep Purif Technol 128:45-57

28. Stephan AM, Teeters D (2003) Electrochim Acta 48:2143-2143

29. Jasiewicz K, Pietrzak R (2013) Sci World J 2013:1-7

30. Mousavi SM, Saljoughi E, Ghasemipour Z, Hosseini SA (2012) Polym Eng Sci 52:2196-2205

31. Hofman M, Pietrzak R (2011) Chem Eng J 170:202-208 